\title{
Mitteilungen und Nachrichten.
}

\author{
Zur Religion der alten Thraker. II. \\ Von Gawril Kazarow.
}

Daß menschliche Eigennamen von Götternamen abgeleitet oder daß Götternamen auf Menschen ohne weiteres übertragen worden sind, ist eine bekannte Tatsache (Usener, Götternamen). Auch die thrakischen Namen, die uns in ziemlich großer Zahl vorliegen ${ }^{1}$ ), machen davon keine Ausnahme. Wir haben gesehen ${ }^{2}$ ), daß Usener mit Recht einen thrakischen Heilgott Darsos rekonstruiert hat; es

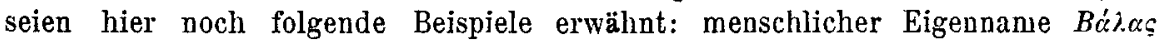

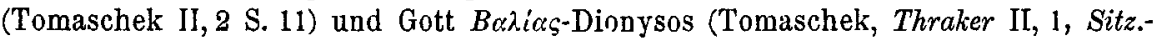
Ber. d. Wien. Akad. 130, S. 41); vgl. auch den Ort Baגoi $\alpha$ in Macedonien (Tomaschek,

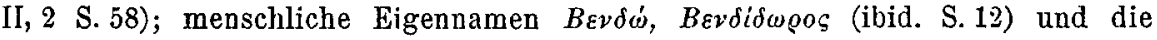

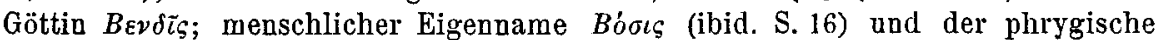
$Z \varepsilon \dot{v} \varsigma$ Bó̧ıos; „dieser phrygische Beiname hat keine geographische Bedeutung “ ${ }^{3}$ );

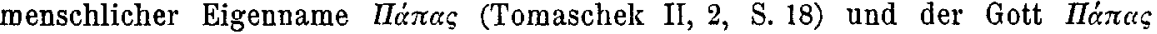
(ibid. II, 1, S. 42); menschlicher Eigenname Manius und der thrakische "Hows

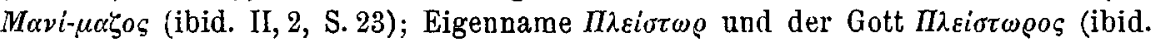

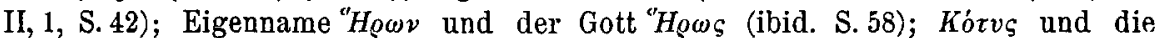

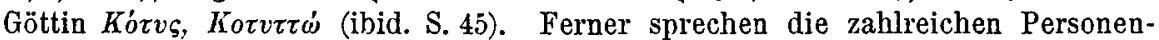
namen mit erstem Bestandteil deo-, dio-, diu-, deos- ${ }^{4}$ ) dafür, daB auch in anderen zusammengesetzten thrakischen Namen sich Götternamen verbergen. ${ }^{5}$ )

Es sei uns gestattet, noch auf Folgendes aufmerksam zu machen. In einer in Thrakien (bei dem Dorf Turkmischli, Bezirk von Cirpan) gefundenen Inschrift (Dobrusky, Sbornik XII, 333) begegnen wir dem Personennamen $\Gamma \varepsilon \rho \mu a v o ́ .^{6}$ ) Dieser zweifelsohne thrakische Name ${ }^{7}$ ), der sich bis auf den heutigen Tag in Bulgarien

1) Seitdem Tomaschek, Die alten Thraker II, 2 (Sitz.-Ber. der Wiener Akad. Bd. 131, 1894), die thrakischen Namen gesammelt hat, sind nicht wenige neue aus Inschriften bekannt geworden, die aber noch nicht zusammengestellt sind.

2) S. Klio, Beiträge zur alten Geschichte Bd. IV, 1. Heft S. 116.

3) Cumunt, bei Pauly-Wissowa R. E. III, 800 s. v. Bozenos.

4) Die Thraker haben einen Himmelsgott dieses Namens verehrt, Kretschmer, Einleitung in die Gesch. der griech. Spr. 241.

5) Beachtenswert ist der Name eines neuen thrakischen Gottes, den wir in einer bei dem Dorfe Aptaat (Bezirk von Dobrič in Dobrudscha) aufgefundenen Inschrift lesen (publiziert von Dobrusky in dem bulgarischen Sbornik des Ministeriums der Volksauflelärung Bd. XVIII, S. 766). Die Inschrift lautet: $\dot{\alpha} . \tau$. $\vartheta \varepsilon \tilde{\omega}(\iota) \Delta \alpha \beta c \xi-$

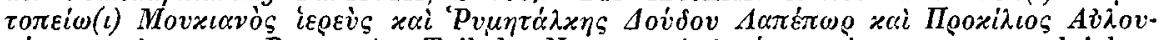

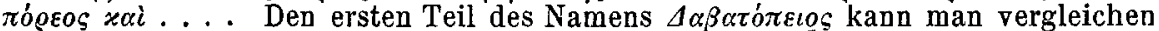
mit den $\Delta \tilde{a ̈ o c ~(l a t . ~ d a ̄ v o s) ; ~ v g l . ~ K r e t s c h m e r, ~ E i n l e i t u n g ~ i n ~ d i e ~ G e s c h . ~ d e r ~ g r i e c h . ~ S p r . ~}$ 214, 221; Brandis bei Pauly-Wissowa, IV, 1949.

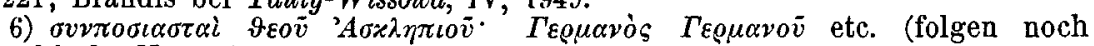
11 thrakische Namen).

7) Vgl. die Ortsnamen Stelu-germane (Tomaschek, II, 2, S. 81), $\Gamma \varepsilon q u \alpha \nu \dot{\eta}$ (ibid. S. 88), heute Saparevska-banja; nicht weit von Sofia liegt das Dorf German, in dessen Nähe sich warme Quellen finden; nicht wenige thrakische Ortsnamen, besonders Gebirgs- und Flußnamen, haben sich bis auf den heutigen Tag erhalten; viele Beispiele bei Tomaschek a. a. 0 . Wir möchten hinzufügen, daß auch der

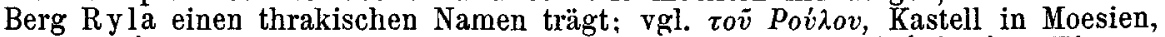

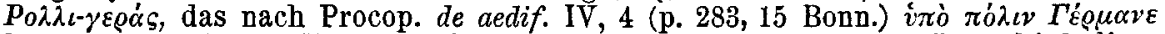
lag, also gerade am Fuße des Ryla (Dobrusky, Sbornik XI, 88). Zwar hieß diese Bergkette im Altertum Joũvas (so richtig Kiepert, Formae orbis antiqui, Illyricum 
erbalten hat, ist, wie wir annehmen möchten. auch Name eines alten thrakischen Gottes gewesen. ${ }^{1}$ ) Es ist beachtenswert, daß dieser Name in bulgarischen Bräuchen eine Rolle spielt, nämlich bei der Regenbeschwörung. *)

Bei Dürre und Regenlosigkeit versammeln sich Mädchen und Burschen und verfertigen eine menschliche Figur von Lehm. Diese Figur, die man German nennt, wird zur Bestattung bereitet mit allen Zeremonien, die bei gewöhnlicher Totenbestattung üblich sind, und die wir hier nicht aufzählen wollen. Nachdem man den German förmlich beweint hat, bringt man ihn zu einem Fluß und begräbt man ihn an dessen Ufer. Manchmal wirft man den German in den FluB. In einigen Ortschaften wird der German, auf eine Tragbahre gestellt, von Haus zu Haus herumgetragen, indem die Begleiter folgendes Lied singen:

Gestorben German an Dürre,

An Dürre, für Regen,

Nimm Grüße mit,

Germane, Germane!

Dabei bekommen die Begleiter von jedem Haus etwas zu essen; davon wird nach vollendeter Bestattung ein Totenmahl bereitet, das zu Ehren des verstorbenen German verzehrt wird.

Es ist nicht dieses Ortes, eine Erklärung des geschilderten Brauches zu geben. Die Vermutnng, daß wir in dem „German “ den alten thrakischen Gott Germanos vor uns haben, ist sehr naheliegend. Dieselbe wird man nicht uuwahrscheinlich finden, wenn man Folgendes in Betracht zieht. Die zahlreichen Inschriften mit thrakischen Namen, die uns erhalten sind, zeigen deutlich, daB die alte thrakische Bevölkerung sich his in späte Zeit, besonders im Lande, in großer Zahl erhalten hat: Diese Thraker sind in die Slaven, die Moesien, Thrakien und Macedonien überschwemmten, aufgegangen und bilden einen integrierenden Teil des heutigen bulgarischen Volkes. ${ }^{3}$ ) Wenn dem so ist, sind wir durchaus berechtigt, zu vermuten, daß thrakische Sitten und Gebräuche, religiöse Vorstellungen noch heute im bulgarischen Volke fortleben, wie es auch mit den Ortsnamen der Fall ist; wir zweifeln nicht, daß die heutige geographische Nomenklatur in Bulgarien viele thrakische Elemente enthält, die zu erkennen wir noch nicht imstande sind; neues epigraphisches Material wird weitere Aufklärung auch auf diesem Gebiete bringen. Und überhaupt zeigt die materielle und geistige Kultur der Bauern in einigen Kreisen Bulgariens frappante Ähnlichkeiten mit der alten thrakischen. ${ }^{4}$ ) Unter diesen Umständen wird Inan unsere Vermutung nicht zu kühn finden. ${ }^{5}$

Freilich, über das Wesen dieses thrakischen Gottes läßt sich vichts sicheres aussagen; wenn wir die Etymologie des Wortes Germanos zu Hülfe nehmen ${ }^{6}$ ), werden wir vermuten dürfen, daß dieser Gott mit der Wärme und der Hitze in

et Thracia; vgl. auch meine Bemerkungen in Wochenschr. f. klass. Pilol. 1905, S. 930flg.), aber das ist, wie es scheint, kein thrakischer Name, nach Tomaschek (a. a. O. S. 89), vielleicht eine bastarnische Benennung; gall. dù-no-“. Nicht unmöglich ist's, daß die umwohnenden Stämme diesen Berg verschieden benannt haben.

1) Das hat schou Bassanovitsch (Sbornik, V, 109) vermutet, ohne freilich zureichende Beweise beizubringen.

2) Über diese Bräuche vgl. A. Stoïlov, Sbornik, Bd. XVIII, S. $641 \mathrm{flg}$.

3) Vgl. H. Schurt\%, Völkerkunde, 1903, S. 145-146.

4) Viele Beispiele bei Bassanovitsch a. a. 0 .

5) Es sei noch erwähnt, daß J. N. Smiruov den alten thrakischen Gott $\Delta \varepsilon o \zeta \varepsilon \lambda \alpha \dot{\alpha} \tau \eta \varsigma$ im heutigen albanesischen Wort dordoléts wiederfindet; der dordoléts steht ebenfalls mit der Regenbeschwörung in Verbindung (Grundriss der Kulturgeschichte der Sïdslaven (russ.) Heft I, S. 37).

6) Zur Etymologie vgl. Kretschmer, a. a.0. S. 231. 
Beziehung stehe, wie es auch im geschilderten bulgarischen Braucle durchschimmert. Wir haben schon bemerkt, dab der Name dieses Gottes auf Personen übertragen worden ist; er findet sich auch in Ortsnamen wieder. ${ }^{1}$ )

\section{$Z_{u}$ den Siedlungsverhältnissen der mykenischen Epoche. Von Ernst Kornemann.}

Da ich in meinem Aufsat\% Polis und Urbs (Klio V $72 \mathrm{ff}$.) auf die vordorische Zeit nicht näher eingegangen bin, möchte ich hier für diese Epoche einen kleinen Nachtrag zu meinen früheren Ausführungen liefern. Die Ausgrabungeu auf Kreta haben ein sehr interessantes Faktum zu Tage gefördert: alle "mykenischen“ Paläste dortselbst, ebenso die größeren Ansiedlungen auf der Insel aus derselben Zeit, wie sie in Gurnia, Palaikastro und anderswo ausgegraben worden sind, entbehren jeglicher Ummauerung, während, wie allbekannt, auf dem griechischen Festland (abgesehen von Orchomenos?) und in Troja das Gegenteil der Fall ist. ${ }^{3}$ ) Dabei zeigen die Paläste des Peloponnes große Verwandtschaft mit den kretischen, allerdings nur den jüngeren, die über älteren mit anderem Unriß und anderer Bauweise errichtet worden sind, wie sich das namentlich in Phaistos nachweisen läßt; ${ }^{3}$ ) allerdings sind die kretischen umfangreicher und komplizierter. Auf alle Fälle handelt es sich aber um Anlagen derselben Zeit.

Wie ist das Problem - Mauerlosigkeit in Kreta, Ummauerung auf dem Festland in einer und derselben Periode - zu lösen? Drerup ${ }^{\ddagger}$ ) meint, Kreta müsse „ein im Innern geeinigtes Reich unter der Herrschaft eines Oberkönigs gewesen sein, da die verschiedenen Fürstensitze der Festungswehr durchaus ermangeln, miteinander also nicht rivalisiert haben können". Diese Annalıme ist zulässig, hat aber keine audere Stütze, als die sagenhafte Überlieferung von der Seeherrschaft des Minos. Dagegen spricht die Tatsache, daß wir heute schon eine ganze Reihe glänzender Herrensitze auf Kreta kennen und unter ihnen zwei (Knossos und Phaistos), die an Ausdehnung einander fast gleichkommen. Allerdings bleibt ja die Möglichkeit bestehen, daß ein Herrscher alle diese Paläste besessen habe, bezw. daß die Inhaber der kleineren Paläste dem Herrn von Knossos untergeordnet gewesen sind. Doch scheint mir auch in diesem Falle das gäuzliche Fehlen jeglicher Verteidigungsmittel um die Paläste und Siedlungen noch nicht hinlänglich erklärt. Einen anderen Deutungsversuch machte Montelius auf dem vorjährigen Archäologen-Kongreb in Athen.5) $\mathrm{Er}$ meinte, aus dem Tatbestand

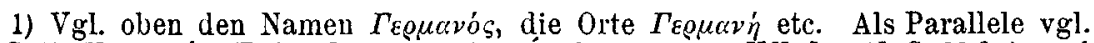
den Gott Kevdoró́s (Reinach, Revue des Études grecques XV [1902] S. 32 fg.) und den Ort Kivdec (Tomaschek, II, 2, S. 85).

2) Auch auf deın Festlande bezieht sich das oben Gesagte anf einzelne Paläste wie auf größere Ansiedlungen, vgl. die Anlage im (damals schon einmal trocken gelegten) Kopaissee (Gla-Paläokastro), Noack, Athen. Mitt. 19, 1894, S. 405 ff., über die Ringmauer spez. 424 ff, Drerup, Homer S. $66 \mathrm{f}$ und-Abbild auf S. 78 und 79 (Nr. 64-65). Abgesehen von der ummauerten Hauptsiedlung befaud sich hier noch eine ganze Reihe kleiner mykenischer Burgen und Befestigungen um den Nordrand des Sees, von wo aus die Verbindungswege nach dem euböischen Mreere beherrscht wurden, Noack 440 ff.; Drerup 67. Über den Befund in Orchomenos vgl. Furtwängler, Berl. phil. Wochenschr. 23 (19. Sept. 1903) Sp. 1213. Hier heißt es allerdings: Auf eine friedliche Herrschaft läßt auch die Tatsache schließen, daß aus minyscher und mykenischer Zeit jede Spur einer Stadtmauer fehlt, ebenso wie in den mykenischen Städten auf Kreta und anscheinend auch in Thessalien". Die abschließende Publikation über die Ausgrabungen in Orchomenos habe ich leider noch nicht zu Gesicht bekommen.

3) Dörpfeld auf dem Interuat. Archäol.-Kongreß zu Athen, vgl. Comptes rendus Z. 10, ausführlicher Ath. Mitt. 30, 1905, S. $257 \mathrm{ff}$.

4) Homer 104; vgl. auch S. 127. 5) Comptes rendus 208. 